\title{
Case Series of Left Stellate Ganglion Blocks for Refractory Angina Pectoris: 14 years later and still efficacious
}

\section{Background:}

Refractory angina pectoris (RAP) is conventionally defined as a chronic pain condition characterized by chest pain resulting from diffuse coronary artery disease which cannot be treated by a combination of optimal medical therapy, or revascularization. In Europe, the annual incidence of RAP is estimated at 30,000-50,000 new cases per year.

Traditional options for this patient group are limited to anti-anginal drug therapy and secondary risk factor modification. Long-term mortality in this patient group was thought to be very high, but recent case series of spinal cord stimulation for RAP showed $50 \%$ survival after implantation of 5 years and the mortality rate among all patients with RAP has been found out to be $<4 \%$ annually; $70 \%$ survive for 9 years or more. It thus becomes important to focus on symptom control and ways to achieve an improved quality of life in this growing population.

Among the reported therapies for pain due to RAP are neuromodulation by transcutaneous electrical nerve stimulation (TENS) or patient-controlled spinal cord stimulation (SCS) and coronary sinus reducers to optimize endo-epicardial blood flow ratio. In 2005, Moore et al published in this journal a trial of temporary left cervical sympathectomy for pain control in RAP.5 They compared this to paravertebral blockade and concluded that cervical sympathectomy is a safe and easy analgesic option. The first publication on sympathectomy to relieve chest pain was in 1933 and the potential for long-standing benefits from intermittent blockade was described in 2000.

We describe the use of cervical sympathectomy via repeated left stellate ganglion block (LSGB) for management of RAP.

\section{Comments:}

Our centre's case series demonstrate how a scientific trial has triggered a useful and minimally invasive treatment for RAP and provided better analgesia for patients who suffer from this debilitating pain condition. We maintain high standards and continue offering this treatment as an outpatient procedure in a department with resuscitation facilities.

\section{Z Tan ${ }^{1}$, J Nalpon², K Valchanov ${ }^{3}$}

${ }^{1}$ Associate Consultant, Department of Anaesthesia and ICU, Singapore General Hospital

${ }^{2}$ Acute Pain Specialist Nurse, Department of Nursing, Royal Papworth Hospital, UK

${ }^{3}$ Consultant, Department of Anaesthesia and ICU, Royal Papworth Hospital, UK

\begin{tabular}{|c|c|c|c|c|c|}
\hline $\mathrm{Pt}$ & $\begin{array}{l}\text { Pain score } \\
\text { before block }\end{array}$ & $\begin{array}{l}\text { Pain score } \\
\text { after block }\end{array}$ & $\begin{array}{l}\text { Pain score } \\
\text { after } 5 \text { wks }\end{array}$ & $\begin{array}{l}\text { Angina attacks + } \\
\text { frequency }\end{array}$ & $\begin{array}{l}\text { Duration } \\
\text { of block }\end{array}$ \\
\hline 1 & $8-10$ & $3-4$ & $5-6$ & $\begin{array}{l}\text { Daily. } \\
\text { Continuous. } \\
\text { All day. }\end{array}$ & $\begin{array}{l}\text { Up till } 8 \\
\text { weeks. }\end{array}$ \\
\hline 2 & $9-10$ & 7 & 8 & $\begin{array}{l}5-7 \text { times a day } \\
\text { Lasts for } 20- \\
25 \text { mins }\end{array}$ & 10 weeks \\
\hline 3 & 6 & 0 & $2-6$ & $\begin{array}{l}\text { Every other day. } \\
\text { Once a day }\end{array}$ & 12 weeks \\
\hline 4 & 8 & 3 & 0 & On exertion & 8 weeks \\
\hline 5 & $7-8$ & $5-6$ & $5-6$ & $\begin{array}{l}\text { Daily angina } \\
\text { attacks } \\
\text { Around 20- } \\
\text { 30mins }\end{array}$ & 12 weeks \\
\hline 6 & 8 & 5 & $5-6$ & $\begin{array}{l}\text { Sometimes twice } \\
\text { a day. Sharp and } \\
\text { short. }\end{array}$ & 10 weeks \\
\hline
\end{tabular}

\begin{tabular}{|c|c|c|c|c|}
\hline $\begin{array}{l}\mathrm{Pt} \\
\text { No }\end{array}$ & Age & Sex & Past Medical History & Interventions \\
\hline 1 & 73 & $M$ & $\begin{array}{l}\text { Housebound } \\
\text { Uses mobility scooter } \\
\text { IHD }\end{array}$ & $\begin{array}{l}\text { CABG } 1997 \\
\text { Trial of SCS } 2007 \\
\text { AF Ablation } 2018\end{array}$ \\
\hline 2 & 67 & $M$ & $\begin{array}{l}\text { AAA } \\
\text { Bladder cancer } \\
\text { Right humeral and left } \\
\text { ankle fracture, IHD }\end{array}$ & $\begin{array}{l}\text { CABG 1986,1997 } \\
\text { Coronary stenting } 2018 \\
\text { SCS } 2004 \\
\text { Reimplantation of SCS } 2007\end{array}$ \\
\hline 3 & 56 & $M$ & $\begin{array}{l}\text { AMI } 2004 \\
\text { Previous apical thrombus }\end{array}$ & $\begin{array}{l}\text { Coronary stenting } 2005 \text {, } \\
2009,2014,2019\end{array}$ \\
\hline
\end{tabular}

Stellate Ganglion Block

First trial in 2007

II weekly basis

(total of 32 LSGB)

First trial 2009

3 monthly basis since $20 \mathrm{II}$

(total of 29 LSGB since 20II)

First trial 2010

3 monthly basis

Declined CABG due to young (total of 28 LSGB since 20I I) age

$4 \quad 48 \quad M \quad$ IHD

$5 \quad 7 I \quad M \quad$ Bilateral TKR IHD

$6 \quad 68 \quad M \quad$ Learning disabilities

$\begin{array}{llll}7 & 62 & M & \text { Morbid obesity }\end{array}$

8 7I M Conn's syndrome HTN, IHD

963 M Refractory angina unknown Coronary Stents 2013 origin

$10 \quad 67 \quad M \quad$ Refractory angina of

$1155 \quad M \quad$ IHD

$1253 \quad M \quad$ Coronary sinus aneurysm OSA, IHD OSA,T2DM

Depression, IHD

SCS 2017- successful unknown origin

\section{CABG 2015}

Coronary stenting 2015

Declined SCS

Coronary stenting 2010

Not suitable for CABG

Declined SCS

Coronary stenting 2017/2018

Coronary stenting

17/2018

First trial 2017

8 weekly basis

(total of 7 LSGB since 2017)

First trial 2017

3 monthly basis

(total of 6 LSGB since 2017)

First trial 2018

(total of 6 LSGB since 2018)

2018

2019

2015

2015

2017

Coronary Stents

CABG 2017

\section{Complications}

7/3/16 No symptom relief, repeated in 2 weeks

20/3/17 No symptom relief, repeated in 2 weeks

$5 / 12 / 16$ No symptom relief, repeated in I week

5/12/16 Neck haematoma

4/12/17 Neck haematoma

16/1/17 No symptom relief, repeated in I week

None

None

None

Short symptom relief <I week

None

Unsuccessful

Unsuccessful

Unsuccessful

Unsuccessful 